\title{
Miranda
}

Revue pluridisciplinaire du monde anglophone /

Multidisciplinary peer-reviewed journal on the English-

speaking world

15 | 2017

Lolita at 60 / Staging American Bodies

\section{Présentation publique du livre de Manon Labry, Pussy Riot Grrrls (Éditions iXe, collection Racine, 2017).}

Librairie Floury, Toulouse, Jeudi 15 juin 2017

Philippe Birgy

\section{(2) OpenEdition}

Journals

Édition électronique

URL : http://journals.openedition.org/miranda/11108

DOI : 10.4000/miranda. 11108

ISSN : 2108-6559

Éditeur

Université Toulouse - Jean Jaurès

Référence électronique

Philippe Birgy, « Présentation publique du livre de Manon Labry, Pussy Riot Grrrls (Éditions iXe, collection Racine, 2017). », Miranda [En ligne], 15 | 2017, mis en ligne le 06 octobre 2017, consulté le 16 février 2021. URL : http://journals.openedition.org/miranda/11108; DOI : https://doi.org/10.4000/ miranda. 11108

Ce document a été généré automatiquement le 16 février 2021.

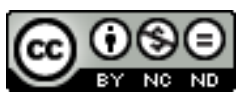

Miranda is licensed under a Creative Commons Attribution-NonCommercial-NoDerivatives 4.0 International License. 


\section{Présentation publique du livre de Manon Labry, Pussy Riot Grrrls (Éditions iXe, collection Racine, 2017).}

Librairie Floury, Toulouse, Jeudi 15 juin 2017

Philippe Birgy

Philippe Birgy

Nous sommes ici pour parler avec Manon Labry de son livre qui vient d'être publié aux Éditions iXe, et dont le titre est Pussy riot grrrls : émeutières. C'est un titre qui combine deux mouvements, pussy riot et riot grrrl, suggérant qu'ils sont liés, et pas seulement consécutifs, avec d'une part beaucoup de bruit de guitares et, d'autre part, une action médiatique, aboutissant à une forme militante commune. Ces deux groupes d'émeutières se retrouvent dans la parole, l'écriture et la performance. Ayant précisé cela, je vais brièvement présenter l'auteur ainsi que son livre afin de pouvoir au plus vite engager un dialogue avec Manon Labry et susciter un échange avec son auditoire. Cette circulation de la parole est au cœur de l'ouvrage, et c'est la motivation première des femmes dont elle raconte l'histoire. 


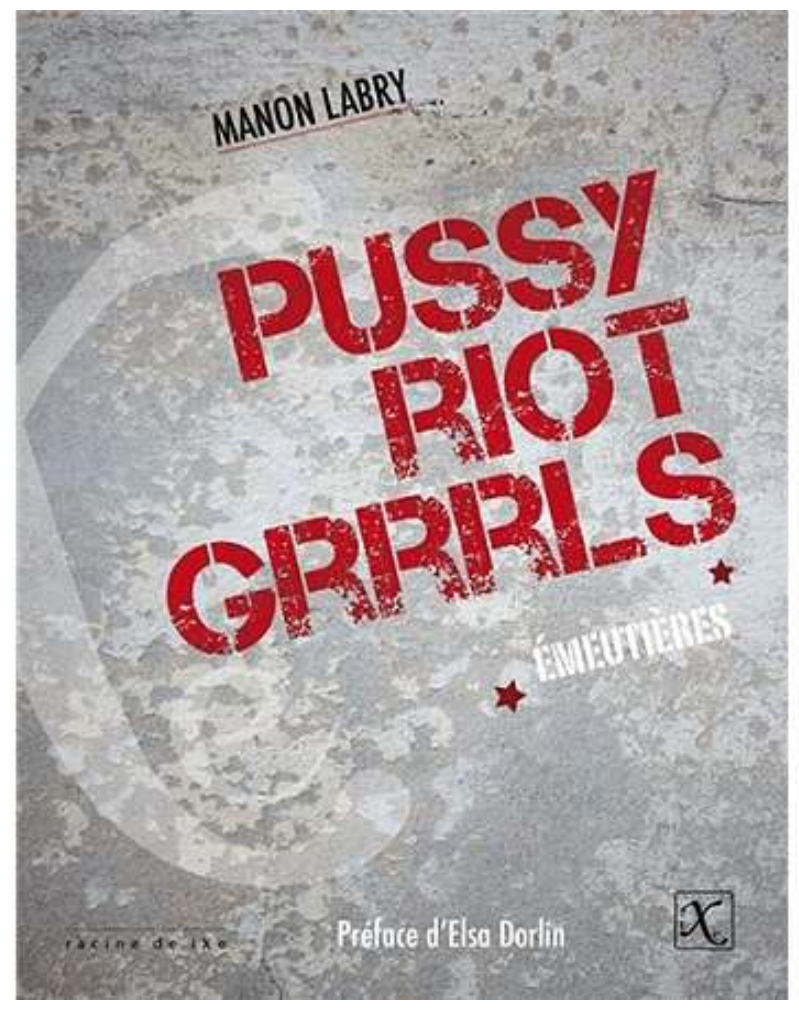

2 Manon a réalisé une thèse sur le cas de la sous-culture punk américaine. Il y a donc un pan universitaire de son travail qui alimente ses écritures plus récentes. Elle a cependant perçu les limites de cet exercice universitaire en ce sens que l'activité de documentation et d'analyse des sources qu'une thèse suppose reste disciplinaire. Même si son travail se trouvait à cheval sur plusieurs disciplines, il s'agissait toujours de décrire les objets d'étude, ce qui les fixe, comme c'est également le cas dans la presse généraliste ou spécialisée, un tel souci du détail pouvant finir par embaumer l'objet qu'on se proposait d'évoquer. À force d'en décrire minutieusement tous les traits, on risque de le figer dans une figure statique. Or, ce qui intéressait Manon Labry dans le Riot Grrrl, c'était davantage son aspect fluide, ouvert, indéterminé et queer, sa dynamique et sa vigueur qui ne se laissaient pas facilement naturaliser ou fossiliser. Il faut ajouter à cette présentation sommaire que Manon Labry mène aussi des activités musicales, ce qui signifie qu'elle a un autre accès pratique à la question de la scène ; nous ne parlons pas ici seulement du simple fait d'être sur scène mais aussi de ce que signifie l'engagement dans une scène.

Quant au livre, Manon Labry dit qu'il y a une manière de traiter le Riot Grrrl comme un style musical, une sorte de phénomène datable et identifiable. Tant du côté de la presse généraliste que des magazines spécialisés, du mainstream que de l'underground, on en a limité la portée en le restreignant à une période et un type de public. Or Manon soutient plutôt l'idée d'une persistance du mouvement, avec des ramifications et des mutations imprévisibles. Elle inscrit les ladyfests dans cette efflorescence. Il ne s'agit pas d'une lignée directe, mais d'une expansion dans toutes les directions, du proche au lointain : il y a les pussy riot, il y a l'electroclash, il y a même une scène techno. La première question serait donc: comment perçois-tu le développement de cet ensemble sur le long terme? Ce qui implique que tu répondes aussi à cette autre question qui peut paraitre simple au premier abord: qu'est-ce que le riot grrrl? Une partie de 
l'auditoire pensera peut-être qu'il est inutile de revenir sur des définitions aussi élémentaires alors que, précisément, il y a peut-être eu trop de simplifications.

Manon Labry

Pour que les choses soient plus claires en ce qui concerne les deux livres - parce que je suis obligée de mentionner qu'il y en a eu un premier sur le sujet -, ce moment de description où l'on a observé un phénomène, un moment d'apparition précis... J'ai dû en passer par là aussi. J'ai dû tomber moi-même dans le piège de la datation quand j'ai écrit Riot Grrrls : Chronique d'une révolution punk féministe. Mais je savais déjà qu'il y aurait un autre livre, et celui dont on parle aujourd'hui porte davantage sur la suite des événements et sur ces ramifications que tu as mentionnées. Il est plus académique.

Pour en revenir à l'origine, le riot grrrl est un mouvement qui est né aux Etats-Unis au tout début des années quatre-vingt-dix, on pourrait même dire à la toute fin des années quatre-vingt. C'est un mouvement culturel, politique, féministe, mené par de jeunes femmes qui ont voulu faire entendre leurs voix dans un milieu où il n'était pas très facile de se faire entendre. Si le punk au départ se voulait égalitaire, horizontal, s'il y a eu des femmes dès le début dans le punk, très vite pendant les années quatre-vingt tout se re-codifie et se re-hiérarchise et le punk devient essentiellement mâle. Et des femmes vivent, chacune dans leur coin, ce sentiment d'aliénation, à la fois dans ces scènes (punk) et dans la société de manière plus générale. Je n'ai pas dit où tout cela se passait : à Olympia, dans l'état du Washington, à une heure de Seattle, une zone qui à cette époque est très dynamique puisque ce sont les débuts du grunge ; et il y a aussi un vivier à Olympia car c'est une ville universitaire. Ces femmes finissent donc par s'identifier les unes les autres, grâce à des fanzines, tels que Jigsaw, qu'on pourrait qualifier de proto-girlzine. C'est le premier qui va permettre à ces femmes de correspondre, de voir qui a envie de faire des choses. Rapidement, elles vont collaborer, fonder d'autres fanzines, monter des groupes et, de fil en aiguille, elles vont aussi concentrer leurs activités sur un autre pôle, à Washington (sur la côte Est, à ne pas confondre avec l'État du Washington), autre site musical très vivant à cette époque-là.

Ce sont deux scènes qui ont un certain nombre d'affinités, qui sont très attachées au Do It Yourself, anticapitalistes, qui ne s'attachent pas à des critères commerciaux, qui font le choix de l'innovation et de l'engagement. Et il y aura beaucoup de déplacements entre ces deux villes de la part des protagonistes de ce premier courant. Elles vont donc se concentrer en même temps sur ces deux pôles. Elles commencent à monter un collectif Riot à Washington en 1991, à organiser des réunions hebdomadaires non mixtes, à créer de nouveaux fanzines. En quelques mois, cela va avoir un effet boule de neige, facilité par les média mainstream, même si par la suite les relations vont devenir un peu conflictuelles. C'est sans doute un peu bref, il s'agit juste de brosser un premier tableau, mais on va peut-être revenir sur les détails plus tard.

Philippe Birgy

On peut évoquer les quelque soucis éditoriaux que tu as connus pour ce livre-ci. Après tout, c'est un problème assez commun que ces délais de publication. Pussy Riot Grrl: émeutières est resté en souffrance pendant un temps assez long, et il aurait normalement dû être publié avant Riot Grrrls: Chronique d'une révolution punk féministe. On y trouve deux tons très différents. Dans le livre publié aux éditions de la Découverte, sorti l'année dernière, tu adoptes la position d'une participante qui essaie de transmettre toute la vitalité, l'enthousiasme, l'exaltation, l'énergie qu'a pu conférer le mouvement à celles qui s'y sont engagées. Et pour ce faire tu te prêtes à un exercice qui 
consiste à raconter l'histoire en la fictionnalisant, en empruntant des anecdotes aux protagonistes du mouvement dont tu parles. L'ensemble de leur activité est un acte d'expression qui passe par une parole publique, par la diffusion de fanzines, et ces femmes-là font leur histoire; elles n'hésitent pas à l'inventer, à exagérer le nombre de magazines existants, et finalement cette fiction donne envie à d'autres femmes de les rejoindre, de rentrer dans le circuit. Il y a donc tout l'héritage du punk, du DIY, que l'on traduit par «bricolage » mais qui a véritablement une autre implication: si quelque chose n'existe pas et que cela manque, il faut le faire soi-même. Et si c'est fait, alors cela rentre dans l'histoire ou la chronique. Cela devient un événement qui aura des conséquences.

Dans tes deux livres, on distingue un phénomène fréquent: d'une part il y a cette émergence d'une scène au même moment, à des endroits différents, sans qu'il y ait de rapport direct (une série de groupes qui définiraient une même orientation esthétique, par exemple), donc un effet d'efflorescence ou plutôt un effet champignon. Et puis cela part dans tous les sens, tu utilises le terme de marcotage, d'une plante qui donne naissance à une série de rejets. Il ne s'agit pas de branches qui partiraient d'un même tronc qui en serait l'origine unique et en déterminerait les qualités communes. On a là quelque chose qui ressemble davantage à ces rhizomes qui courent sous la terre et qui vont faire qu'une repousse sortira vingt mètres plus loin. Tu utilises aussi la métaphore de la contagion : tout cela se répand comme un virus.

Manon Labry

L'une des idées qui est vraiment au cœur de la démarche des riot grrrls, c'est ce slogan « revolution girls style now ", la révolution à la manière des filles maintenant. Je vous lis un extrait d'un de leurs manifestes: "nous jugeons que la fantasmagorie de la révolution permanente macho porte-flingue n'est qu'un mensonge irréalisable destiné à nous laisser rêver plutôt qu'à incarner notre rêve. Et donc nous cherchons la révolution dans nos propres vies, chaque jour, en imaginant et en créant des alternatives à la manière merdique chrétienne et capitaliste de faire les choses ».

Il y a donc cette idée que la révolution qu'elles proposent n'est pas du tout l'idée qu'on s'en fait normalement. C'est au contraire une succession et une addition de révolutions individuelles. Elles passent par la prise de parole, par la création, par le DIY et c'est encore plus révolutionnaire pour les femmes qu'on incite davantage à se taire. L'idée cruciale, c'est que ce n'est pas un courant qui va tenter de fixer ses contours, mais il va les flouter pour essayer de le rendre accessible et transformable. La question de la prise de parole est cruciale et elle va entraîner ce dont tu parlais. Le mouvement va être repris par des gens qui vont se reconnaitre comme ses héritiers et se le réapproprier dans d'autres contextes, sous d'autres formes qui se réinventent constamment comme les ladyfest, les girls rock camps, les pussy riot. Tout cela participe de l'idée de contagion lente, d'une révolution patiente, qui s'inscrit dans la durée et qui signifie que, par la création, la personne qui s'est investie a fait sa propre révolution.

11 Philippe Birgy

Il y a un prix à payer pour cela, qui est qu'il faut accepter que cela disparaisse temporairement, que ces petits foyers restent sporadiques. Et dans ta conclusion tu parles d'Hakim Bey et de sa notion de Zones Temporaires d'Autonomie. Ces vingt minutes de présentations peuvent peut-être donner une première idée de ce que le texte de Manon Labry peut receler de réflexions stimulantes. Je pense qu'on s'était entendu sur l'idée de faire circuler la parole et que le moment est propice. 
on Labry

Pendant les premiers moments d'émergence de cette culture, la première moitié des années quatre-vingt-dix, le mouvement a concerné très majoritairement les États-Unis, le Canada, et un peu l'Angleterre. En France, il y a le problème des archives qui se pose. À ma connaissance, il n'y avait pas en France des collectifs aussi organisés qu'aux ÉtatsUnis. Ce n'est qu'avec internet que cette culture est devenue accessible, à la fin des années quatre-vingt-dix, à toute une population qui était totalement passée à côté, qui était trop jeune aussi pour avoir connu ses précédents. Mais c'est déjà la fin de ce premier moment d'émergence. En France, ce qui a très bien fonctionné, ce sont les ladyfests. En 2000, toujours à l'initiative des personnes qui avaient fait partie des premiers moments, a été organisé un festival ladyfest destiné à promouvoir une culture féministe DIY, un festival où il y avait à la fois des performances, des ateliers, des groupes de discussion. C'était donc un événement qui proposait des moments de distraction, mais aussi des moments sérieux d'élaboration d'une espèce de théorie de la pratique, de mise en commun des savoirs et des savoir-faire. Et tout cela a été créé avec l'idée que ça devait circuler. Les organisatrices ont clairement annoncé : vous qui êtes là, prenez l'idée et faites-en quelque chose dans vos communautés, dans les lieux ou vous résidez. Et c'est ce qui s'est passé. En France, il y en a eu une dizaine. Des collectifs informels qui partageaient cette culture riot grrrl se sont montés. Le concept a assez bien pris car deux ou trois cents ladyfests se sont tenus en une quinzaine d'années, entre 2000 et 2015 , dans le monde entier.

$\mathrm{Tu}$ as mentionné la question de la documentation qui est très importante. Tu es spécialiste du riot grrl et je ne me risquerais pas sur ce terrain, mais je peux citer l'exemple du punk. Depuis un certain nombre d'années des démarches sont entreprises pour retrouver les traces des initiatives punk. C'est un autre aspect de l'histoire culturelle populaire: d'un côté on la fait, mais de l'autre il est important de la mémorialiser, sans quoi on aboutit à une sorte de chronique héroïque très centralisée où le punk serait l'initiative d'une poignée de jeunes gens à Londres en 1976. Or il y avait au même moment, sur la côte Ouest des États-Unis, des groupes qui adoptaient sensiblement la même approche dès 1975. On recense les groupes, en France, en Espagne et au Portugal, qui portaient le même projet, et cela change évidemment la perception d'ensemble que l'on a du mouvement. Il est donc capital de collationner toutes ces informations, de retrouver la trace des enregistrements autoproduits, des fanzines éphémères qui assuraient la liaison entre les membres de telle ou telle scène régionale ou locale.

15 Manon Labry

C'est quelque chose qui m'avait beaucoup marqué au sujet des riot grrls. Il y avait finalement peu de matériaux disponibles au moment où j'ai commencé à travailler sur le sujet. Et au fur et à mesure que les publications sortaient sur ce sujet, je m'apercevais que ça se figeait. L'idée était donc de trouver des éléments qui n'avaient pas été mentionnés, de les raconter d'une autre manière, ou de proposer une approche très subjective, de dire : voilà ce que je pense, au-delà d'un strict récit des faits. C'était une manière de dire "voilà ce que moi j'en ai tiré ", en contournant ces fixations. J'ai eu la chance que se crée le fonds d'archives de New York, à NYU, un fonds rassemblé par une 
archiviste qui avait elle-même participé aux premiers ladyfests, et à laquelle une vingtaine de personnes avaient donné l'ensemble de leurs archives. Cela inclut un petit flyer griffonné taché par le café, des objets anodins qui permettent d'écrire une histoire différente, et qui n'est pas figée.

Question du public

Est-ce que les pussy riot ont changé quelque chose dans le mouvement ?

Les pussy riot sont un cas très particulier, une réinvention totale, le contexte n'est pas le même. Cela n'a pas la même portée politique, et ce n'est pas le même usage des médias non plus. Ce sont des gens qui se sont autant inspirés des riot grrls que de l'artivisme moscovite. Avant elles, il n'y a pas vraiment d'occurrence de liens avec les milieux de l'art contemporain. Quand elles ont été emprisonnées, un nombre énorme de collectifs se sont formés dans le monde entier, souvent sous le nom "free pussy riot ", des gens ont organisé des manifestations, des soirées de soutien pour récolter des fonds et cela redonné un souffle à des activités militantes car comme tu le disais, il faut accepter qu'il y ait des baisses de régime, que parfois il n'y ait plus rien, comme c'est le cas en ce moment, par exemple. Les pussy riot ont redonné une certaine vigueur au mouvement pendant une année.

Question du public

Est-ce qu'on a des données sur la diffusion du mouvement dans le reste du monde ?

Il y a eu des collectifs riot grrl en Malaisie et au Japon. Il y a aussi eu beaucoup de ladyfest en Amérique du Sud. C'est sans doute ce qui a le mieux marché car il y avait l'idée que plus cela se diffuserait, plus le mouvement serait enrichi par de nouvelles influences, de nouvelles musiques.

Ce qui a également bien marché, ce sont les girls rock camps, en Asie, en Amérique du Sud. Je n'ai plus les détails en tête mais vous les trouverez dans le livre. Cela se passe sensiblement à la même époque que les ladyfests. Tout part de Portland, où une femme a l'idée de monter des sortes de colonies de vacances inspirées des ladyfests pour des jeunes filles de sept à dix-sept ans, avec l'objectif de les initier à jouer en groupe. Qu'elles soient musiciennes ou pas, cela n'avait pas d'importance. Il s'agissait non seulement d'apprendre à jouer d'un instrument et d'apprendre à jouer en groupe, mais de s'initier à la composition. L'idée était de travailler une chanson et de la jouer au terme de la colonie de vacances. Et dans le même temps, il y avait aussi des ateliers d'auto-défense, de culture féministe, tous adaptés à l'âge des participantes. Et ces camps ont eu un succès phénoménal, il y en a eu une cinquantaine aux États-Unis, mais aussi en France, en Angleterre, en Suède, en Autriche, en Islande, au Brésil et au Japon.

Question du public

À part l'état des lieux, est-ce qu'on peut identifier des acquis, des choses qui ont fait bouger les lignes socialement?

\section{Manon Labry}

Il est difficile de répondre, puisque c'est un mouvement qui oscille sans cesse entre l'individu et le collectif, les effets réels sont individuels, on peut très difficilement le quantifier. Ce que l'on peut remarquer, ce sont des changements de comportements dans les milieux underground, des attitudes qui étaient tolérées il y a encore quinze ans ne le sont plus du tout. 
INDEX

Mots-clés : culture populaire, ladyfests, punk, performance, queer

Thèmes : Music

Keywords : popular culture, ladyfests, punk, performance, queer

\section{AUTEURS}

PHILIPPE BIRGY

Professor à l'UT2J

birgy@univ-tlse2.fr 